\title{
ANÁLISE SWOT COMO FERRAMENTA PARA AVALIAÇÃO DA AGROBIODIVERSIDADE EM SISTEMAS TRADICIONAIS DE PRODUÇÃO NOS MUNICÍPIOS DE ANTONINA E MORRETES/PR
}

\section{SWOT ANALYSIS AS A TOOL FOR ASSESSING THE AGROBIODIVERSITY IN TRADITIONAL PRODUCTION SYSTEMS IN THE MUNICIPALITIES OF ANTONINA AND MORRETES / PR}

\author{
Adriana de BIASSIO \\ Ivan Crespo SILVA ${ }^{1}$
}

\section{RESUMO}

Este estudo buscou avaliar a agrobiodiversidade e sua função mantenedora da atividade agrícola/florestal praticada nas áreas estudadas. Para isso foram avaliados 20 sistemas produtivos, cujos tamanhos compreenderam de 1 a 3 módulos fiscais. As avaliações foram feitas por meio de observações diretas no campo e entrevistas aos agricultores, onde foram aplicados questionários semi-estruturados. Para a avaliação dos resultados foi realizado a análise SWOT para identificação dos fatores críticos de sucesso dos sistemas produtivos identificados nas áreas. Com relação à análise SWOT, os fatores críticos de sucesso externos revelaram que as oportunidades são superiores as ameaças identificadas, significando que a agricultura familiar deve ser incentivada na região; e os fatores críticos de sucesso internos indicam que a região de estudo mostra-se adequada para o desenvolvimento das atividades rurais, sendo necessário realizar melhorias no sistema produtivo para torna-se mais produtivo e rentável. Para a superação dos pontos negativos listados é necessário que os agricultores busquem formas de diversificação de suas propriedades e juntamente com os órgãos responsáveis sejam desenvolvidos opções que atendam as necessidades e realidades locais, oferecendo cursos de capacitação para o fortalecimento da agricultura orgânica e/ou industrialização dos produtos e incentivo ao turismo rural.

Palavras-chave: Agrobiodiversidade, Análise SWOT e Políticas Públicas.

\section{ABSTRACT}

This study aims to evaluate the agrobiodiversity and its function maintains the agriculture/forestry practiced in the areas studied. 20 were evaluated for this production systems, with sizes comprised 1 to 3 tax modules. Evaluations were made by means of direct observations in the field and interviews with farmers, which were applied semi-structured questionnaires. For the evaluation of the results was carried out a SWOT analysis to identify the critical success factors of production systems in the areas identified. With regard to the SWOT analysis, the critical success factors revealed that the external opportunities outweigh the threats identified, meaning that family should be encouraged in the region, and the critical success factors indicate that the internal study area proved to be adequate development of rural activities, being necessary to make improvements in the production system to become more productive and profitable. To overcome the drawbacks listed is necessary for farmers to seek ways to diversify their properties and along with the bodies responsible options are developed that meet local needs and realities, offering training courses to strengthen the organic farming and / or manufacturing products and encouraging rural tourism.

Keywords: Agrobiodiversity, SWOT Analysis and Public Policy. 


\section{INTRODUÇÃO}

A agrobiodiversidade ou biodiversidade agrícola diz respeito a todas as espécies de plantas, animais e microorganismos interagindo dentro do agroecossistema, com o propósito de produzir alimentos e matérias-primas para a indústria de vestuário, farmacêutica, construção civil, mobiliários e afins (Armando, 2002). Ela pode ser compreendida como parcela utilizada da biodiversidade, representada por um conjunto de organismos e ecossistemas que apresentam fortes relações com os seres humanos (Figura 1), podendo ser domesticados, semi-domesticados, cultivados, ou manejados pelo homem (Stella, 2006).

Um estudo realizado sobre agrobiodiversidade e tecnologia em sistemas produtivos de campesinos foi observado que os agricultores estão interessados em maximizar a produtividade de diversas espécies, essa estratégia é resultado da baixa produtividade do trabalho devido aos métodos artesanais e heterogeneidade ambiental, podendo afirmar que a agrobiodiversidade é também resultado do tipo de tecnologia utilizada na exploração da terra e condições do processo produtivo (Cáceres, 2006).

No Brasil, as áreas dos agricultores familiares estão normalmente em ambientes marginais, onde a agricultura caracteriza-se por complexas variações nas condições agroecológicas e socioeconômicas, sujeitos a complexos estresses e a elevados custos e riscos de produção (Machado; Machado, 2007).

A Análise SWOT é uma ferramenta estratégica para organização e planejamento muito utilizada na área administrativa para a qual foi originalmente criada. A SWOT é usada para detectar possíveis ameaças e oportunidades (fatores externos), fortalezas e fraquezas (fatores internos), determinar atividades e contribuir na definição de objetivos; sua realização possibilita mostrar "o que deve ser feito" para melhorar os sistemas produtivos (Antonio, 2002).

A SWOT fornece informações quanto às oportunidades a serem aproveitadas, sobre quais ameaças serão enfrentadas e os pontos fracos que devem ser minimizados ou neutralizados para meIhorar o desempenho do sistema (Ansoff; Mcdonnell, 1993). Depois da identificação dos pontos fortes e fracos, ameaças e oportunidades quando respondidas pelos envolvidos é possível ter uma meIhor visão do sistema tanto interna quanto externamente (Houben et al., 1999).

Devido à falta de informações específicas a respeito da agrobiodiversidade na região estudada, o presente estudo buscou determinar os fatores internos (forças e fraquezas) e fatores externos (oportunidades e ameaças) que podem influenciar positivamente e negativamente na manutenção das atividades agrícolas e florestais estudadas.

\section{MATERIAIS E MÉTODOS}

A área de estudo localiza-se na região do sul do Brasil, no Estado do Paraná, situada na planície litorânea. O litoral paranaense representa 3\% da área total do estado, composto por sete municípios (Borsatto, 2007). O estudo foi desenvolvido em 20 propriedades rurais, sendo 10 em cada município, localizadas em Antonina e Morretes, entre julho de 2010 a maio de 2011. As localidades foram escolhidas por contemplarem predominantemente pequenas propriedades rurais, de unidade familiar as quais foram selecionadas para o presente estudo. O motivo da escolha prende-se a falta de informações relacionadas de agrobiodiversidade desse estrato de produtores rurais.

Foram realizadas visitas às propriedades e feitas entrevistas aos produtores rurais. As seleções das propriedades obedeceram aos seguintes critérios de seleção:

propriedades cujo tamanho compreende de 1 a 3 módulos ficais, sendo que cada módulo fiscal possui 16 hectares, de acordo com o INCRA (2001); propriedades representativas da agricultura familiar praticada na região.

Foram selecionados agricultores na região de Santa Fé, Rio Sagrado, Pantanal, Rio Pequeno, Ponte Seca, Pitinga, Central, Sapitanduva, Sapiá de Baixo, Passa Sete e margens de estrada em Morretes, no Bairro Alto, Rio Pequeno, Estrada da Barra, São João, Cacatu, Turvo e margens de estrada em Antonina.

As entrevistas foram feitas por meio de questionário semi-estruturado. $O$ questionário foi desenvolvido, conforme Oliveira (2006), para buscar informações sobre fraquezas, fortalezas, ameaças e oportunidades relativas aos sistemas de produção praticados, de maneira a atender os preceitos da análise SWOT.

Como referência para as entrevistas utilizou-se um informante qualificado, em geral representado pelo proprietário ou "chefe" de família, que no caso foi predominantemente homem, conforme tradição do meio rural local. Em alguns casos da ausência masculina o informante qualificado foi à mulher. Os resultados obtidos das entrevistas foram organizados e analisados através de estatística descritiva, considerando a freqüência, a consistência e a coerência das respostas.

A matriz SWOT foi estruturada com dados sobre vantagens e desvantagens dos sistemas produtivos e identificação dos seus pontos fortes e fracos, bem como das oportunidades e ameaças relacionadas pelos agricultores.

Como pressuposto para a análise SWOT as fortalezas e fragilidades foram definidas como perspectivas que dependem somente do agricultor $e$ seu sistema produtivo.

As oportunidades e ameaças, por sua vez, foram definidas como atividades que independem da ação do agricultor e de resposta de seus 
sistemas produtivos, mas sim de fatores externos, como, p.ex., aspectos da legislação, ações inerentes a governo, burocracias, política pública, assistência técnica entre outros.

Após registrar e listar as informações, estas foram agrupadas para cada município formando um ranking com base nas informações mais recorrentes em cada grupo.

As informações foram na seqüência estruturadas em uma matriz (matriz SWOT), em conformidade com Montana e Charnov (2003). Após a elaboraçao da matriz SWOT e apartir desta, visando reduzir a subjetividade da análise SWOT, construiu-se outras matrizes para avaliação quantitava de fatores considerados externos (MFE) e internos (MFI) que influenciam no comportamento e organização do sistema.

Para a aplicação da MFE e MFI seguiuse os procedimentos recomendados por David (1998), a seguir:

Para a listagem dos fatores críticos de sucesso, foi primeiro agrupadas as oportunidades e depois as ameaças;

Determinação de um peso entre 0,00 (considerado não importante) à 1,00 (muito importante), para cada fator listado. O peso indicará a importância do fator na obtenção de sucesso, sendo que sua soma total deve ser igual a 1,00.

Pontuação de cada fator crítico externo/interno de 1 à 4 , para indicar a efetividade do sistema de cultivo em responder a condição de sucesso. Critérios:

4 - maior significância, resposta superior;

3 - acima da média;

2 - resposta média;

1 - resposta pobre.

$\mathrm{Na}$ sequência, multiplicou-se cada fator pelos pelo valor recebido;

Somou-se os resultados obtidos para obtenção do total de pontos do sistema avaliado. Quanto mais alto o resultado maior a capacidade do sistema em aproveitar as oportunidades e reduzir ameaças.

De acordo com David (1998), independente da quantidade de fatores que são incluídos na MFE e MFI, o ranqueamento total varia de 1,0 a ponderação máxima de 4,0 , com pontuação média ponderada de 2,5. Quando a soma dos escores ponderados forem menores que 2,5 o sistema será considerado como fraco em relação ao aproveitamento das oportunidades e mais sujeito as influências negativas das ameaças identificadas (TABELA 1).

Tabela 1 - Classificação de fatores internos do sistema produtivo a partir do valor ponderado total.

\begin{tabular}{|c|l|}
\hline Valor ponderado & \multicolumn{1}{|c|}{ Fatores internos } \\
\hline$<2$ & Não apta \\
\hline $2-3$ & Moderadamente apta \\
\hline $3-4$ & Apta \\
\hline$>4$ & Muito apta \\
\hline
\end{tabular}

Da mesma maneira, procedeu-se em relação aos fatores internos dos sistemas produtivos, estabelecendo-se a Matriz para Valoração dos Fatores Críticos Internos (MFI) com a identificação de fortalezas e fragilidades dos sistemas avaliados.

\section{RESULTADOS E DISCUSSÕES}

Para a análise de SWOT foram consideradas características que podem influenciar negativamente ou positivamente nos sistemas produtivos das áreas avaliadas. Estas informações conforme (David, 1998) foram classificadas em quatro grupos: fortalezas, fraquezas, oportunidades e ameaças (Tabela 2).

Dos pontos listados foram extraídos os fatores críticos de sucesso, de maior influência no sistema produtivo.

As fortalezas e fraquezas são pontos que estão relacionados com a capacidade e influência dos fatores que podem contribuir ou prejudicar os sistemas produtivos encontrados. Esses aspectos foram relacionados a partir de observações de campo e das respostas obtidas durantes as entrevistas com os agricultores.
Em relação às oportunidades e ameaças, foram considerados os aspectos que podem influenciar os sistemas produtivos, mas que tem origem externa a eles, em geral que estão na dependência de ações políticas ou de outros acontecimentos que fogem ao controle do agricultor.

As oportunidades foram classificadas de acordo com a sua importância em relação a sustentabilidade do sistema produtivo, enquanto que as ameaças, pelo contrário, com a importância negativa que podem exercer. Os dois aspectos oportunidades e ameaças foram em conformidade com Kother (2000).

Para os produtores as oportunidades foram definidas como aspectos que estes acreditam importantes para gerar melhoria no sistema em reação a diversificação de renda e escoamento da produção. Já as ameaças foram relacionadas como aspectos que representam riscos para a manutenção do sistema de produção. Um curioso exemplo de ameaça foi citado como a dificuldade de licenças ambientais, a qual pode inviabilizar ou atrasar as atividades que precisam ser realizadas sob o ponto de vista do agricultor. 
BIASSIO, A.; SILVA, I.C. Análise SWOT...

Os fatores internos da SWOT geraram uma matriz com valores quantitativos, sendo identificadas nesta as fortalezas e fraquezas dos sistemas produtivos (Tabela 3 ). Os totais encontrados nessa pontuação foram de 2,80 para critérios de sucesso interno e 2,94 para critérios de sucesso externo, esses valores encontram-se acima da média ponde- rada, citada com valor em 2,5. Valores abaixo disso, indicam que o sistema não é viável para ser adotados por outros produtores rurais. (David, 1998). Como este valor encontra-se pouco acima da média, pode ser um indicativo da necessidade de meIhorias no sistema, para que ele seja viável e esteja apto para ser adotado por outros agricultores.

Tabela 3- Matriz para valoração dos fatores críticos internos (MFI)

\begin{tabular}{lccc}
\hline FATORES CRÍTICOS DE SUCESSO & PESO & DESEMPENHO & RESULTADO \\
\hline \multicolumn{1}{c}{ FORTALEZAS } & & 0,16 \\
Segurança; qualidade de vida; & 0,04 & 4 & 0,18 \\
$\begin{array}{l}\text { Industrialização da matéria prima para agregação de va- } \\
\text { lor; }\end{array}$ & 0,06 & 3 & 0,4 \\
$\begin{array}{l}\text { Enriquecimento do sistema com espécies de valor econô- } \\
\text { mico; }\end{array}$ & 0,1 & 4 & 0,06 \\
$\begin{array}{l}\text { Produção orgânica; } \\
\text { Diversificação de espécies cultivadas e da propriedade; }\end{array}$ & 0,06 & 1 & 0,3 \\
Maquinário próprio; & 0,1 & 3 & 0,08 \\
Venda de polpa da Juçara. & 0,06 & 1 & 0,12 \\
\hline
\end{tabular}

\section{FRAQUEZAS}

Dificuldade de acessos as propriedades;

Terrenos ondulados, solos fracos;

Baixos preços praticados para produtos agrícolas;

Falta de valoração de produtos orgânicos;

Pragas e animais silvestres prejudicam as plantações;

Falta de informações e assistência técnica;

Dependência do aluguel de máquinas para plantio.

$\begin{array}{ccc}0,1 & 4 & 0,4 \\ 0,08 & 2 & 0,16 \\ 0,08 & 4 & 0,32\end{array}$

$\begin{array}{lll}0,04 & 1 & 0,04\end{array}$

$\begin{array}{lll}0,02 & 2 & 0,04\end{array}$

$\begin{array}{lll}0,08 & 3 & 0,24\end{array}$

$\begin{array}{lll}0,1 & 3 & 0,3\end{array}$

\begin{tabular}{llll}
\hline Total & 1,00 & - & 2,80 \\
\hline
\end{tabular}

Os fatores críticos externos estão apresentados na Tabela 4 compostos pelas principais oportunidades e ameaças dos sistemas produtivos.

As fortalezas identificadas foram: a possibilidade da prática da venda direta para prefeituras, feiras, bancas localizadas na beira das estradas e comércios da região; buscar o cultivo de plantas aromáticas, medicinais e ornamentais na região; formação de mercado para produtos orgânicos possibilitando a melhoria da qualidade de vida e a renda dos agricultores, a busca pela diversificação da propriedade, cursos de aperfeiçoamento e orientação técnica para execução das atividades específi- cas que atendam as demandas e necessidades dos agricultores rurais e incentivo ao turismo rural.

As fraquezas ou ameaças que mais perturbam os sistemas são a falta de acesso as propriedades, pois isso prejudica a logística para entrada de insumos e escoamento da produção, assim como o acesso dos produtores a cidade; e prejuízos com as pragas, em especial o "caramujo africano", que ataca mudas e sementes dos cultivos praticados. O caramujo africano tem hábito noturno e é de difícil controle (Fisher, 2006).

Os resultados encontrados, a partir de médias ponderadas, com valores entre 2 e 3 , classi- 
ficam os sistemas como moderadamente aptos em conformidade com David (1998).

Resultados semelhantes foram encontrados por Salzmann (2009), que comparou dois sistemas agroflorestais um localizado em Cerro Azul PR e outro em Dali - China, reafirmando a metodo-

Tabela 4 - Matriz para valoração dos fatores críticos externos (MFE)

\begin{tabular}{|c|c|c|c|}
\hline FATORES CRÍTICOS DE SUCESSO & PESO & DESEMPENHO & RESULTADOS \\
\hline \multicolumn{4}{|c|}{ OPORTUNIDADES } \\
\hline $\begin{array}{l}\text { Venda direta, sem atravessadores } \\
\text { (lanchonetes, mercados; feiras); }\end{array}$ & 0,1 & 4 & 0,4 \\
\hline $\begin{array}{l}\text { Mercado de aromáticas, medicinais e ornamen- } \\
\text { tais; }\end{array}$ & 0,04 & 1 & 0,04 \\
\hline $\begin{array}{l}\text { Formação de mercado para produtos orgâni- } \\
\text { cos; }\end{array}$ & 0,04 & 1 & 0,04 \\
\hline $\begin{array}{l}\text { Formação de mercado para diferentes produ- } \\
\text { tos agrícolas; }\end{array}$ & 0,08 & 3 & 0,24 \\
\hline Cursos de aperfeiçoamento; & 0,08 & 2 & 0,16 \\
\hline Incentivo ao turismo rural. & 0,08 & 3 & 0,24 \\
\hline \multicolumn{4}{|c|}{ AMEAÇAS } \\
\hline Falta de rodovias adequadas; & 0,12 & 4 & 0,48 \\
\hline $\begin{array}{l}\text { Falta de conhecimento de fluxos de caixa; } \\
\text { (entrada e saída de mercadorias) }\end{array}$ & 0,08 & 3 & 0,24 \\
\hline $\begin{array}{l}\text { Falta assistência da EMATER/IAPAR/ } \\
\text { Prefeitura }\end{array}$ & 0,08 & 2 & 0,16 \\
\hline $\begin{array}{l}\text { Burocracias para licenças em órgãos ambien- } \\
\text { tais e prefeituras }\end{array}$ & 0,12 & 4 & 0,48 \\
\hline $\begin{array}{l}\text { Ocorrência de enchentes em épocas de chu- } \\
\text { vas. }\end{array}$ & 0,1 & 3 & 0,3 \\
\hline $\begin{array}{l}\text { Desinteresse da nova geração com as ativida- } \\
\text { des agrícolas. }\end{array}$ & 0,08 & 2 & 0,16 \\
\hline Total & 1,00 & & 2,94 \\
\hline
\end{tabular}

logia proposta por David (1998) e utilizada no presente estudo. Em relação aos sistemas produtivos identificados pode-se inferir que ações externas a eles em especial no âmbito de políticas publicas podem favorecer o desenvolvimento e a sustentabilidade dos mesmos.
Venda direta, sem atravessadores

tais;

Formação de mercado para produtos orgâni-

Formação de mercado para diferentes produ-

tos agrícolas

Ações como a manutenção das rodovias demais vias de acesso às propriedades, fortalecimento da dinâmica da venda direta em feiras, intensificação de programas municipais de merenda escolar, além de assistência técnica e exigências burocráticas para retirada de licenças em órgãos ambientais, podem efetivamente favorecer os sistemas produtivos identificados.

Segundo Houben et al. (1999) o bom funcionamento de um sistema é o resultado da interação de ambos os ambientes: interno e externo, sendo importante concentrar os objetivos e decisões futuros, levando em consideração as fortalezas, e evitar tendências relacionadas às fraquezas, conforme evidenciado na análise SWOT.

\section{CONCLUSÕES}

- A agrobiodiversidade presente nas áreas avaliadas e os bens e serviços daí resultantes não são capazes, por si só, de garantir sua existência ao longo do tempo. Fontes extras de recursos são ne- cessárias para garantir a sustentabilidade dos sistemas agrícolas em Antonina e Morretes;

- Conforme a análise SWOT, a avaliação e relativização das fortalezas e fraquezas dos sistemas de produção evidenciam que as atividades produtivas podem ser mantidas e incrementadas. Para isto, medidas devem ser tomadas para garantir que os pontos positivos identificados neutralizem ou superem os negativos.

- A análise dos fatores externos que influenciam os sistemas produtivos regionais, em relação ao potencial de oportunidades e ameaças, revela que as oportunidades são superiores as ameaças, significando que a agricultura familiar deve ser incentivada na região.

- Para a superação das ameaças e aproveitamento das oportunidades os agricultores devem buscar formas de diversificação da renda de suas propriedades, seja através do turismo rural, fortalecimento da agricultura orgânica e/ou agroindustrialização dos seus produtos. 
BIASSIO, A.; SILVA, I.C. Análise SWOT...

\section{REFERÊNCIAS}

1. AGROSOFT BRASIL. Diversificação das lavouras de café com a fruticultura melhora renda do agricultor. Agrosoft, abril de 2011. Disponível em: <http://agrosoft.com/pdf.php/?node=217247>. Acesso em 9 de abril de 2011.

2. ANSOFF, H. I; MCDONNELL, E. J. Implantando a administração estratégica. 2 ed. São Paulo: Atlas, 1993. 590p.

3. ANTÓNIO, N. S. Estratégia organizacional: uma evolução nos últimos 50 anos, 2002. Disponível em: <http:// ee.dcg.eg.iscte.pt/estrategia\%20organizacional.pdf>. Acesso em: 10 de maio de 2010.

4. ARMANDO, M., S. Agrodiversidade: ferramenta para uma agricultura sustentável. Documentos 75. Brasília: Embrapa Recursos Genéticos e Biotecnologia, 2002. 23 p.

5. BASTARDZ, C. Análise da preferência da paisagem do município de Morretes, Paraná como subsídio ao planejamento do turismo. Dissertação (mestrado), Universidade Federal do Paraná, 2009. 123p.

6. BORSATO, R. S. Agroecologia um caminho multidimensional para o desenvolvimento agrário do litoral Paranaense. Dissertação (Mestrado em Agronomia). Universidade Federal do Paraná. Curitiba. 2007.

7. CACERES, D. M. Agrobiodiversidade y tecnologia em sistemas productivos campesinos. jun., vol.31, n.6, 2006. p.403410. Disponível em: <http://www.scielo.org.ve/scielo.php?script=sci arttext\&pid=S0378$18442006000600005 \&$ Ing=es\&nrm=iso\&tlng=en>. Acesso em: 9 de novembro de 2010.

8. DAVID, F. R. Strategic Management: concepts \& cases. 7 ed. New Jersey. EUA. Prentice Hall Inc, 1998.

9. DOSSA, D. MONTOYA, L. J. A atividade florestal e agroflorestal como alternativas de renda aos produtores rurais. Circular técnica, n. 56, novembro, Colombo, EMBRAPA Florestas, 2001. 6p.

10.FABRE, M. Produção intransitável. Folha de Londrina, Rural, 26 de março de 2011. Disponível em: <http://200.175.180.15/webclipping/openantt/noticia.php?pg=6\&id_cliente=75\&id_edicao=2\&data=26/03/2011>. Acesso em: 26 de março de 2011.

11.FAO - Building on Gender, agrobiodiversity and local knowledge - A training manual, 2005. $177 \mathrm{p}$

12.FISCHER, M. L; SIMIÃO, M; COLLEY, E; ZENNI, R. D; SLVA, D. A.T; LATOSKI, N. O caramujo exótico invasor na vegetação nativa em Morretes, PR: diagnóstico na população de Achatina fulica Bowdich, 1822 em um fragmento de floresta ombrófila densa aluvial. Biota neotropica, v. 6, n. 2, maio- agosto, 2006. 5p.

13.GRISE, M. M. A estrutura da paisagem do mosaico de unidades de conservação do litoral do Paraná. Dissertação (mestrado), Universidade Federal do Paraná, 2008. $120 \mathrm{p}$

14. HOUBEN, G., LENIE, K., VANHOOF, K. A knowledge-based SWOT-analysis system as an instrument for strategic planning in small and medium sized enterprises. Decision Support Systems, 26, 1999. p. 125-135.

15.INCRA. Tabela do sistema nacional de cadastro rural - índices básicos de 2001, 2001. Disponível em:<www.jacto.com.br/ portarias/INCRA.xls.> Acesso em: 12 de abril de 2011

16.KOTHER, P. Administração de marketing: a edição do milênio. São Paulo: Prentice Hall, 2000. 764 p.

17. MACHADO, A. T; MACHADO, C. T. T. Melhoramento participativo de cultivos no Brasil. In: BOEF, W. S.; THIJSSEN, M. H.; OGLIARI, J. B.; STHAPIT, B. R. Biodiversidade e agricultores: fortalecendo o manejo comunitário. Porto Alegre: L\&PM, 2007. p. 93-102.

18.MDA - Ministério do Desenvolvimento Agrário. Pronaf: Programa Nacional de Fortalecimento da Agricultura Familiar. Disponível em: <http://portal.mda.gov.br/portal/saf/programas/pronaf/2259636>. Acesso em: 12 de abril de 2011.

19.MONTANA, P. J; CHARNOV, B. H. Administração. 2 ed. São Paulo: Saraiva, 2003. 525p.

20.NYCHA, L.; SOARES, A. C. C. S. A relação do processo agroindustrializante e a pequena propriedade rural: prospecções para o desenvolvimento local/regional. In: Anais do V Encontro de Economia Paranaense - ECOPAR. Curitiba-PR 2007.

21. OLIVEIRA, R. C. Uso e manejo dos recursos nos arredores das residências de camponeses - Estudo de caso na região da Morraria, Cáceres - MT. 166 f. Dissertação (mestrado em agricultura tropical). Universidade Federal do Mato Grosso, Cuiabá, 2006

22.PELINSKI, A; AHRENS, D.C; MILLÉO R. D. S; ZEMKE, E; BERNASSI, D. A; RICHTER, A. S. A diversificação do incremento da renda da propriedade familiar agroecológica. Instituto Agropecuário do Paraná, 2006. Disponível em: <http:// www.iapar.br/arquivos/File/zip_pdf/Trab011Diversif.pdf>. Acesso em: 1 de abril de 2011.

23.RAUCH, P. SWOT analyses and SWOT strategy formulation for forest owner cooperation in Austria. European Journal Forest Research, 126. 2007. p. 413

24. RICHETTI, A. O que é diversificação agropecuária? Portal do agronegócio, 2006. Disponível em: < http:// www.portaldoagronegocio.com.br/conteudo.php?id=23258 >. Acesso em: 1 de abril de 2011.

25.SCHIERE, H. Perda da diversidade e de raças de animais domésticos: um tema esquecido. In: BOEF, W. S.; THIJSSEN M. H.; OGLIARI, J. B.; STHAPIT, B. R. Biodiversidade e agricultores: fortalecendo o manejo comunitário. Porto Alegre: L\&PM, 2007. p. 53-66.

26.SCHNEIDER, S. A Pluriatividade na Agricultura Familiar. Porto Alegre: UFRGS - Universidade Federal do Rio Grande, RS, 2003. 253p.

27.SILVA SOUZA, L. O turismo rural: instrumento para o desenvolvimento sustentável. Edição eletrônica. Disponível em: $<$ www.eumed.net/libros/2006c/194/>. Acesso em: 14 de abril de 2011.

28.STELLA, A.; KAGEYAMA, P. Y.; NODARI, R. Políticas públicas para a agrobiodiversidade. In: Agrobiodiversidade e diversidade cultural. Brasília, DF: MMA, 2006. $82 \mathrm{p}$

29.VIANA, G; RINALDI, R. N. A. Principais fatores que influenciam o desempenho da cadeia produtiva de leite: Um estudo com os produtores de leite do município de Laranjeiras do Sul, PR. Organizações rurais \& Agroindústrias. Lavras, v.12, ${ }^{\circ}$ 2, 2010. p. 263-274 